\title{
Toward a Case Definition of Adverse Health Effects in the Environs of Industrial Wind Turbines: Facilitating a Clinical Diagnosis
}

Bulletin of Science, Technology \& Society 3I (4) 316-320

(C) 20II SAGE Publications

Reprints and permission: http://www. sagepub.com/journalsPermissions.nav DOI: |0.1 |77/02704676| |4|5075

http://bsts.sagepub.com

@SAGE

\author{
Robert Y. McMurtry'
}

\begin{abstract}
Internationally, there are reports of adverse health effects (AHE) in the environs of industrial wind turbines (IWT). There was multidisciplinary confirmation of the key characteristics of the AHE at the first international symposium on AHE/IWT. The symptoms being reported are consistent internationally and are characterized by crossover findings or a predictable appearance of signs and symptoms present with exposure to IWT sound energy and amelioration when the exposure ceases. There is also a revealed preference of victims to seek restoration away from their homes. This article identifies the need to create a case definition to establish a clinical diagnosis. A case definition is proposed that identifies the sine qua non diagnostic criteria for a diagnosis of adverse health effects in the environs of industrial wind turbines. Possible, probable, and confirmed diagnoses are detailed. The goal is to foster the adoption of a common case definition that will facilitate future research efforts.
\end{abstract}

\section{Keywords}

case definition, clinical diagnosis, wind turbines, adverse health effects, symptoms

\section{Introduction}

On the last 3 days of October 2010, a groundbreaking meeting was held in the Waring House situated in Prince Edward County, Ontario (Society for Wind Vigilance, 2010). The focus of the symposium was the emerging issue of adverse health effects (AHEs) being experienced by people living in the environs of industrial wind turbines (IWTs).

These health effects appear to correlate with proximity to IWTs, the sound pressure level emitted by the IWTs, the frequency of the noise, the time of exposure, and individual response. The pattern of individuals' complaints demonstrates a striking similarity internationally in media reports and in physician-generated case series.

The issue of AHEs is of considerable complexity and has excited much controversy between proponents of the wind industry and those who have identified widespread media and Internet reports of AHEs in virtually all countries where IWTs have been erected (Gray, 2010; Jopson, 2010; Lam, 2009; Turkel, 2010).

The IWT proponents claim IWTs to be a promising green, clean, and free alternative source of electrical power and an ideal solution for reducing green house gases (Canadian Wind Energy Association, 2011; Nextera Energy Resources, 2010). Those who are concerned about IWT development too close to residences and who seek to prevent AHEs have a contrary view denying the foregoing claims and questioning the utility and safety of IWTs (Bryce, 2010; Gilligan, 2010).

This article will concentrate on the health aspects and the challenge of a case definition, leaving aside the debate surrounding economics, energy policy, lobbying, and social marketing, although all have a significant impact on government decision making.

\section{Overview of Conference and Speakers}

The purpose of the symposium was to promote a multidisciplinary dialogue on possible AHEs in an effort to advance the understanding of the genesis of complaints appearing globally. Among the goals of the symposium was a need to develop a case definition, which had been under discussion since June 2010.

The symposium attracted a multidisciplinary international group of speakers (14), including the disciplines of medicine (four specialties), acoustics, psychology, business, physics,

\footnotetext{
'St. Joseph's Health Care, London, Ontario, Canada
}

Corresponding Author:

Robert Y. McMurtry, 3469 County Rd I3, Picton, Ontario, Canada KOK 2T0

Email: Robert.McMurtry@sjhc.london.on.ca 
epidemiology, policy analysts, pharmacy, law, statistics, and media (Society for Wind Vigilance, 2010). There was also an informal research meeting of the speakers joined by two family physicians and an occupational health physician where a debriefing of the symposium was held and future plans for research made.

Approximately 100 people attended the symposium including municipal and federal politicians, media, documentary filmmakers, as well as two members of a leading consulting group for the industry and two representatives from a wind power developer. There was a notable absence of any representatives from the Ontario provincial government.

\section{Brief Summary of Presentations}

The descriptions of the presentations below are highly abbreviated. The reader is referred to the Society for Wind Vigilance's website for more details.

Physics of IWTs and the resultant sound pressure level (SPL) are not adequately or consistently regulated. Based on experience with other noise sources, SPL clearly presents a health risk (Harrison, 2010; James, 2010; Walsh, 2010).

The human ear is perturbed by IWTs in quiet rural areas, potentially leading to neural remodeling and disorganization of neural pathways. It is more likely than not that the symptoms and signs associated with wind turbine syndrome are due to the sound energy emitted by IWTs. Low-frequency noise and infrasound will more likely than not be shown in subsequent research to be playing a major role in the genesis of wind turbine syndrome (Pierpont, 2010).

The outer hair cells of the cochlea respond to low frequency and infrasound. Sonic energy that is inaudible is perceived though not necessarily heard (except in sensitive people). What cannot be heard therefore may produce AHEs. This statement was made by Dr. Alex Salt, referring to his research using the standard animal model (guinea pig) for the study of human hearing (Salt, 2010).

Noise and infrasound during the day are capable of causing mood disorder, cognitive dysfunction, and learning and developmental problems in children. Stress and psychological distress are established findings of chronic exposure to noise. Chronic stress has serious physiological consequences (Bronzaft, 2010).

Nighttime noise compromises restorative sleep. Restorative sleep is a necessary condition for maintaining health and wellbeing. Chronic sleep disturbances (increased arousals and awakenings) and/or deprivation are established AHEs known to substantially increase the risk for chronic disease and premature death (Hanning, 2010).

Control studies comparing populations living near and far from IWT installations demonstrate a substantial and statistically significant difference in quality of life, mood disorders, and sleep disruption (Nissenbaum, 2010).

More than a hundred people in Ontario have self-identified as having AHEs using the Canada Vigilance protocol. AHEs with a very wide range of complaints were made, of which the most frequent are compromise of quality of life, sleep disruption, some living in the environs of IWTs leaving their homes temporarily or permanently in order to restore their health (Krogh, 2010). While some improvement in health status is achieved, follow-up has revealed that preexposure health status is not necessarily regained.

These findings are significant from a public health perspective for many reasons, including the findings the crossover and revealed preference in the WindVOiCe survey (Krogh et al., 2011). Crossover refers to the phenomenon of exacerbation and amelioration when near and far from wind farms, respectively. Revealed preference describes the act of leaving one's accustomed residence permanently or temporarily for significant periods of time in order to achieve restoration.

Legally there is evidence that the precautionary principle has not been respected by the governments who regulate and approve IWT installations in the absence of medical or health evidence establishing their safety (Gillespie, 2010). There is an urgent need to pursue research establishing dose-response curves as well as clinical research regarding psychological and physiological consequences (Bronzaft, 2010; Hanning, 2010).

There was a clear consensus among the foregoing presentations and from a wide variety of perspectives that AHEs are indeed occurring in relationship to people living in the environs of IWTs. In addition, an emerging consensus was evolving regarding a case definition that could be deployed by experts representing the many diverse disciplines in attendance. The importance of unifying the case definition for the purposes of research and future communications was clear.

\section{Audience Response}

The symposium featured a learned and diverse group of speakers as noted above. Attendees were able to witness and participate in a successful event of transdisciplinarity. Regardless of discipline, a unity of perspective was achieved. AHEs are clearly an issue for people living in the environs of wind farms. While the precise mechanism for the cause of AHEs remains to be elucidated, there is enough evidence to conclude IWTs represent a public health threat. Audience members were also highly supportive of a unified case definition.

\section{Summary}

The common denominator of the global reports of AHEs is the compromise of quality of life, restorative sleep, and psychological well-being.

There are many reports of AHEs in the environs of IWTs, including several case series (Harry, 2007). Unfortunately, no standard protocol for data gathering has been developed. This has lead to a wide variety of symptoms being reported and documented. This variance is exacerbated by the nonspecific nature of the complaints since the recorded symptomatology can arise from a wide variety of ailments and diseases. 
The task of a case definition is to weight the unique elements of AHE/IWT to distinguish the clinical disorder from competing explanations. There are common themes found in the reports that are reflected in the first- and second-order criteria. There are few, if any, alternate explanations for the first- and second-order criteria other than AHE/IWT.

The third-order criteria serve the purpose of capturing the most commonly reported symptoms.

It is hoped that future reports will adopt a standardized protocol based on this case definition, which would facilitate future research and management of AHE/IWT.

\section{Case Definition}

The criteria for making an individual diagnosis of probable AHEs in the environs of IWTs are presented in the following paragraphs. The definition endeavors to be specific and sensitive. While the definition has not been validated formally in practice, it has proven useful. The case definition represents an important starting point for future international research collaboration. The genesis of the definition is based on a review of the literature and direct experience with those individuals experiencing AHE/IWT. It has been used to provide guidance to physicians and other primary health providers when they are asked to manage individuals following exposure to IWTs. The value of this proposal is based on the absence of a specific case definition either in the peer-reviewed or gray literature.

\section{Diagnosis of Adverse Health Effects in the Environs of Industrial Wind Turbines}

Possible adverse health effects. Report of a change in health status by people living within $5 \mathrm{~km}$ of a wind farm installation. Further confirmation is required to validate or exclude AHE/IWT by establishing a medical history that satisfies the criteria identified under "Probable Adverse Health Effects" below.

Probable adverse health effects.

1. First-order criteria (all four of the following must be present):

(a) Domicile within $5 \mathrm{~km}$ of industrial wind turbines (IWT)

(b) Altered health status following the start-up of, or initial exposure to, and during the operation of, IWTs. There may be a latent period of up to 6 months

(c) Amelioration of symptoms when more than $5 \mathrm{~km}$ from the environs of IWTs

(d) Recurrence of symptoms upon return to environs of IWTs within $5 \mathrm{~km}$

2. Second-order criteria (at least three of the following occur or worsen after the initiation of operation of (WT): (a) Compromise of quality of life

(b) Continuing sleep disruption, difficulty initiating sleep, and/or difficulty with sleep disruption

(c) Annoyance producing increased levels of stress and/or psychological distress

(d) Preference to leave residence temporarily or permanently for sleep restoration or well-being

3. Third-order criteria (at least three of the following occur or worsen following the initiation of IWTs):

(i) Otological and vestibular
(a) Tinnitus
(b) Dizziness
(c) Difficulties with balance
(d) Ear ache
(e) Nausea

(ii) Cognitive

(a) Difficulty in concentrating

(b) Problems with recall or difficulties with remembering significant information

(iii) Cardiovascular
(a) Hypertension
(b) Palpitations
(c) Enlarged heart (cardiomegaly)

(iv) Psychological

(a) Mood disorder, that is, depression, anxiety

(b) Frustration

(c) Feelings of distress

(d) Anger

(v) Regulatory disorders

(a) Difficulty in diabetes control

(b) Onset of thyroid disorders or difficulty controlling hypo- or hyperthyroidism

(vi) Systemic
(a) Fatigue
(b) Sleepiness

Confirmed adverse health effects. The confirmation of AHE/ IWT is achieved by a clinical evaluation and physiological monitoring of individuals during exposure to IWT sonic energy or an accurate facsimile (recording or other imitative source of IWT sound). Ideally, sleep studies should be carried out in the home of people experiencing AHEs. The complex physiological monitoring equipment required for a sleep study is not readily made mobile. Accordingly, sleep studies need to be carried out in an established clinical sleep laboratory with a source of sonic energy that accurately reflects the person's exposure to IWTs.

The process may be simpler once controlled studies comparing possible victims with a nonexposed matched population are carried out. These studies could help determine the core physiological change(s) that is (are) likely occurring to those who live in the environs of IWTs.

The need to rule out alternate explanations is the responsibility of the licensed clinician. While adherence to the criteria has resulted in no false positive diagnosis to date further validation is required. 


\section{Differential Diagnosis}

Consideration should be given to other stressors present in the community. The most obvious is the wind itself, which when associated with substantial barometric changes is known to cause a variety of symptoms. In this case, the onset of AHEs would not correlate with the establishment of a wind farm nor would the AHEs improve when leaving the environs of a wind farm.

A second possibility is a stressful home environment, which might lead to restoration being more likely away from home. A history for family stressors should be elicited and ruled in or out. Another distinguishing feature is the absence of correlation with IWTs starting up or being in operation.

Psychological issues and/or mood disorders may be simultaneously or independently present. A key differentiating point is the timing of the onset and the impact of being away from home and the environs of IWTs. Significant improvement away from the environs of wind turbines and revealed preference for sleeping away from home serve to distinguish between AHEs due to IWTs versus an independent cause. If the situation appears more complex then a referral to a clinical psychologist or psychiatrist might be considered.

Apart from the foregoing, there are very few if any imitative AHEs that can meet the three orders of criteria outlined above. However, the author invites critical commentary that might indicate a different conclusion.

\section{Conclusions}

1. A multidisciplinary symposium was held to address the possibility of adverse health effects in the environs of industrial wind turbines.

2. There was a consensus (unanimity) among the various experts that more likely than not, adverse health effects are occurring in the environs of industrial wind farms.

3. A case definition for adverse health effects in the environs of industrial wind turbines has been proposed based on the best available evidence. To date it has proven useful in clinical practice.

4. Further research is required to refine and validate the proposed definition and identify the simplest method by which to diagnose a confirmed case.

\section{Declaration of Conflicting Interests}

The author declared no potential conflicts of interest with respect to the research, authorship, and/or publication of this article.

\section{Funding}

The author received no financial support for the research, authorship, and/or publication of this article.

\section{References}

Bronzaft, A. (2010, October). Children: Canaries in the coal mine. Paper presented at the First International Symposium on Adverse Health Effects, "The Global Wind Industry and Adverse Health Effects: Loss of Social Justice?" Picton, Ontario, Canada. Retrieved from http:/www.windvigilance.com/internationalsymposium/proceedings-first-international-symposium

Bryce, R. (2010, March 1). The brewing tempest over wind power. The Wall Street Journal. Retrieved from http://online.wsj.com/ article/SB10001424052748704240004575085631551312608 .html?mod=WSJ_Opinion_LEFTTopOpinion\#printMode

Canadian Wind Energy Association. (2011, April). Wind Facts. Number 5: Environmental benefits. Retrieved from http://canwea .ca/wind-energy/windfacts_e.php

Gillespie, E. (2010, October). Social justice and the law. Paper presented at the First International Symposium on Adverse Health Effects, "The Global Wind Industry and Adverse Health Effects: Loss of Social Justice?" Picton, Ontario, Canada. Retrieved from http:/www.windvigilance.com/international-symposium/ proceedings-first-international-symposium

Gilligan, A. (2010, September 12). An ill wind blows for Denmark's green energy revolution. The Telegraph. Retrieved from http://www.telegraph.co.uk/news/worldnews/europe/ denmark/7996606/An-ill-wind-blows-for-Denmarks-greenenergy-revolution.html

Gray, L. (2010, March 6). Noise complaints about one in six wind farms. Daily Telegraph. Retrieved from http:/www.telegraph .co.uk/earth/earthnews/7377641/Noise-complaints-about-one-insix-wind-farms.html

Hanning, C. (2010, October). Wind turbine noise and sleep. Paper presented at the First International Symposium on Adverse Health Effects, "The Global Wind Industry and Adverse Health Effects: Loss of Social Justice?" Picton, Ontario, Canada. Retrieved from http://www.windvigilance.com/international-symposium/ proceedings-first-international-symposium

Harrison, J. (2010, October). It's pure physics. Paper presented at the First International Symposium on Adverse Health Effects, "The Global Wind Industry and Adverse Health Effects: Loss of Social Justice?" Picton, Ontario, Canada. Retrieved from http://www.windvigilance.com/international-symposium/ proceedings-first-international-symposium

Harry, A. (2007, February). Wind turbines: Noise and health. Retrieved from http://www.windturbinesyndrome.com/news/wpcontent/uploads/2011/02/wtnoise_health_2007_a_harry_noPW .pdf

James, R. (2010, October). How we got here. Paper presented at the First International Symposium on Adverse Health Effects, "The Global Wind Industry and Adverse Health Effects: Loss of Social Justice?" Picton, Ontario, Canada. Retrieved from http://www.windvigilance.com/international-symposium/ proceedings-first-international-symposium

Jopson, D. (2010, April 2). Tilting at windmills: Why families are at war. The Sydney Morning Herald. Retrieved from http:// 
www.smh.com.au/environment/energy-smart/tilting-at-windmillswhy-families-are-at-war-20100401-ri4p.html

Krogh, C. (2010, October). A gross injustice. Paper presented at the First International Symposium on Adverse Health Effects, "The Global Wind Industry and Adverse Health Effects: Loss of Social Justice?" Picton, Ontario, Canada. Retrieved from http://www.windvigilance.com//international-symposium/ proceedings-first-international-symposium

Krogh, C., Gillis, L., \& Kouwen, N. (2011). WindVOiCe (Wind Vigilance for Ontario Communities). A self-reporting survey: Adverse health effects, industrial wind turbines (IWT), and the need for vigilance monitoring. Retrieved from http:// www.windvigilance.com/international-symposium/proceedingsfirst-international-symposium

Lam, T. (2009, September 27). Homeowners fight against the wind; turbines blow ill wind for some. Detroit Free Press. Retrieved from (cited June, 2011) http://knowwind.org/webdoc/ homeowners.htm

Nextera Energy Resources. (2010). Conestogo Project. Retrieved from http://www.canadianwindproposals.com

Nissenbaum, M. (2010, October). Deleterious health effects are undeniable. Paper presented at the First International Symposium on Adverse Health Effects, "The Global Wind Industry and Adverse Health Effects: Loss of Social Justice?” Picton, Ontario, Canada. Retrieved from http://www.windvigilance.com/international-symposium/proceedings-first-international-symposium

Pierpont, N. (2010, October). Defining a syndrome. Paper presented at the First International Symposium on Adverse Health Effects, "The Global Wind Industry and Adverse Health Effects: Loss of Social Justice?" Picton, Ontario, Canada. Retrieved from http://www.windvigilance.com/international-symposium/ proceedings-first-international-symposium

Salt, A. (2010, October). Infrasound: Your ears hear it but they don't tell your brain. Paper presented at the First International Symposium on Adverse Health Effects, "The Global Wind Industry and Adverse Health Effects: Loss of Social Justice?" Picton, Ontario, Canada. Retrieved from http:/www.windvigilance.com/internationalsymposium/proceedings-first-international-symposium

Society for Wind Vigilance. (2010, October). First International Symposium on Adverse Health Effects, "The Global Wind Industry and Adverse Health Effects: Loss of Social Justice?" Picton, Ontario, Canada. Retrieved from http://www.windvigilance .com/international-symposium/proceedings-first-internationalsymposium

Turkel, T. (2010, January 24). Turbines turn into headache for Vinalhaven; Noise complaints energize opponents of wind power and complicate Maine's renewable energy efforts. Portland Press Herald. Retrieved from http://www.istockanalyst.com/article/ viewiStockNews/articleid/3806588

Walsh, O. (2010, October). No global standards. Paper presented at the First International Symposium on Adverse Health Effects, "The Global Wind Industry and Adverse Health Effects: Loss of Social Justice?" Picton, Ontario, Canada. Retrieved from http:/www.windvigilance.com/international-symposium/ proceedings-first-international-symposium

\section{Bio}

Robert Y. McMurtry is the former Dean of Medicine for the University of Western Ontario. He was a member of the Health Council of Canada for $3 \frac{1}{2}$ years and a member and special advisor to the Royal Commission under Roy Romanow on the future of health care in Canada. Dr. McMurtry was a visiting Cameron Chair to Health Canada for providing policy advice to the Minister and Deputy Minister of Health. He was the Founding and Associate Deputy Minister of Population \& Public Health, Canada. Dr. McMurtry also sat on the National Steering Committee on Climate Change and Health Assessment. Presently Dr. McMurtry is Professor (Emeritus) of Surgery, University of Western Ontario. 